Edinburgh 2000/02

hep-ph/0003035

\title{
Treatment of Heavy Quarks in Deeply Inelastic Scattering
}

\author{
Michael Krämer ${ }^{1}$, Fredrick I. Olness ${ }^{2}$, and Davison E. Soper ${ }^{3}$ \\ ${ }^{1}$ Department of Physics and Astronomy, University of Edinburgh, Edinburgh EH9 3JZ, \\ Scotland \\ ${ }^{2}$ Department of Physics, Southern Methodist University, Dallas, Texas 75275, USA \\ ${ }^{3}$ Institute of Theoretical Science, University of Oregon, Eugene OR 97403, USA
}

\begin{abstract}
We investigate a simplified version of the ACOT prescription for calculating deeply inelastic scattering from $Q^{2}$ values near the squared mass $M_{H}^{2}$ of a heavy quark to $Q^{2}$ much larger than $M_{H}^{2}$.
\end{abstract}




\section{Introduction}

The inclusion of heavy quark effects in deeply inelastic scattering (DIS) is an interesting theoretical problem involving two hard scales in a perturbative analysis. This issue is also of phenomenological importance. The charm contribution to the total structure function $F_{2}$ at small $x$, at HERA, is sizeable, up to $25 \%$. Through the charm contribution to scaling violations, the treatment of charm also has a significant impact on the interpretation of fixed target deeply inelastic scattering data. Thus, a proper description of charm contributions to deeply inelastic scattering is required for a global analysis of structure function data and a precise extraction of the parton densities in the proton.

At scales $Q \lesssim M_{H}$, the contribution to deeply inelastic scattering of a heavy quark of mass $M_{H}$ can be calculated in the so-called fixed-flavor-number (FFN) prescription from hard processes initiated by light quarks $(u, d, s, \ldots)$ and gluons, where all effects of the heavy quark $(H)$ are contained in the perturbative coefficient functions. This prescription incorporates the correct threshold behavior, but for large scales, $Q \gg M_{H}$, the coefficient functions at higher orders in $\alpha_{s}$ contain potentially large logarithms $\ln ^{i}\left(Q^{2} / M_{H}^{2}\right)$, which may need to be summed [1, 2, 3]. Such a summation can be achieved by including the heavy quark as an active parton in the proton. The simplest approach incorporating this idea is the so-called zero-mass variable-flavor-number (ZM-VFN) prescription, where heavy quarks are omitted entirely below some scale $Q_{0} \approx M_{H}$ and included as massless partons above this threshold. This prescription has been used in global analyses of parton distributions for many years, but it has an error of order $M_{H}^{2} / Q^{2}$ and is not suited for quantitative analyses unless $Q \gg M_{H}$.

Considerable effort has been devoted to including heavy quark effects in deeply inelastic scattering in such a way that the calculated structure functions match those of the FFN prescription in the region $Q \approx M_{H}$ while they match those of the ZM-VFN prescription for $Q \gg M_{H}$. Two prescriptions of this sort, the Aivazis-Collins-Olness-Tung (ACOT) [4] and the Thorne-Roberts [5] prescriptions have been used in recent global analyses of parton distributions [6, 7]. More recently, additional variable-flavor-number prescriptions with non-zero mass have been defined in the literature [8]. If one could sum perturbation theory, the calculated structure functions should be identical for any prescription that does not neglect the heavy quark mass. However, the way of ordering the perturbative expansion is not unique, so that the results generally differ at any finite order in perturbation theory.

In this paper, we will investigate a modification of the ACOT prescription advocated by Collins [9]. It has the advantage of being simple to state and of allowing relatively simple calculations. This simplicity should be convenient for phenomenological analyses at the Born level. In addition, it could be crucial for implementing a variable-flavor-number prescription with non-zero mass at next-to-leading order in global analyses of parton distributions. 


\section{Schemes and partons}

We consider the structure function $F_{2}(x, Q)$. The observable structure function can be written in terms of parton distribution functions $f$ and a calculable partonic structure function $\hat{F}_{2}$ as

$$
F_{2}(x, Q)=\int_{x}^{1} d \xi \sum_{a} f_{a / p}(\xi, \mu) \widehat{F}_{2}\left(a, x / \xi, \mu / Q, \alpha_{s}(\mu)\right)+\mathcal{O}\left(\Lambda^{2} / Q^{2}\right) .
$$

This factorization formula has corrections of order $\Lambda^{2} / Q^{2}$, where $\Lambda$ is a typical scale of hadronic physics, perhaps the mass of the rho meson. Here $\mu$ is the renormalization/factorization scale. (For simplicity, we do not distinguish these two scales.) The partonic $\hat{F}_{2}$ has a perturbative expansion in powers of $\alpha_{s}(\mu)$. There is a sum over parton types $a=g, u, \bar{u}, d, \bar{d}, \ldots$, and there is a function $f_{a / p}$ for each parton.

To begin, we must pick a scheme for the definition of $\alpha_{s}$ and for the definition of the parton distribution functions. In fact, we will use multiple schemes, following the prescription of [9]. Each scheme is designated by the number $N$ of "active" quark flavors. Scheme $N$ is designed to be most useful for physical scales $Q$ in the range $M_{N} \lesssim Q \lesssim M_{N+1}$, where $M_{N}$ is the mass of the $N$ th quark flavor.

We define what we mean by the running coupling $\alpha_{s}^{N}(\mu)$ in scheme $N$ by defining how we perform renormalization. We renormalize using the CWZ prescription [10]. Briefly, divergences involving active parton loops are removed with an $\overline{\mathrm{MS}}$ subtraction, but when active parton external lines couple to a loop containing "non-partonic" lines (quarks $N+$ $1, N+2, \ldots)$ the renormalization is by subtraction at zero external momentum. The running of $\alpha_{s}^{N}(\mu)$ is controlled by the usual $\overline{\mathrm{MS}}$ renormalization group equation with the contributions from quarks $1, \ldots, N$ in the beta function.

In the scheme $N$ there are parton distributions for gluons and for quark flavors $1, \ldots, N$, but not for quark flavors $N+1, \ldots$. The parton distribution functions are defined to be proton matrix elements of certain operators [1]. The operator products are ultraviolet divergent and are renormalized according to the CWZ prescription. They obey a renormalization group equation - the usual DGLAP equation - in which contributions from quark flavors $N+1, \ldots$ do not appear in the kernel.

Note that the parton distribution functions are non-perturbative objects. There is no question of neglecting any masses in the definition. The quark masses do not appear in the kernel of the evolution equation, but this is because renormalization counter terms are mass independent, not because the parton distributions themselves are mass independent.

Note also that by using CWZ renormalization throughout, quark loops for the nonpartonic flavors decouple from calculations when the momentum scale is small compared to $M_{N+1}$. Thus for $Q \ll M_{N+1}$ it is a good approximation to leave the non-partonic flavors out of calculations altogether. (But leaving heavy flavors out of calculations is not part of the definition of scheme $N$ as used here; it is a separate approximation.) When one does leave the non-partonic flavors out of calculations, the remaining renormalizations are via the $\overline{\mathrm{MS}}$ 
prescription. Thus one commonly refers to parton distributions in the prescription described here as $\overline{\mathrm{MS}}$ parton distributions.

There is a perturbative connection between schemes $N$ and $N+1$ which we can represent by:

$$
f_{a / p}^{N+1}(x, \mu)=f_{a / p}^{N}(x, \mu)+\sum_{b} \int_{x}^{1} \frac{d \xi}{\xi} A_{a b}\left(x / \xi, \mu / M_{N+1}, \alpha_{s}(\mu)\right) f_{b / p}^{N}(\xi, \mu) .
$$

Here the index $a$ runs over $g$, the $N$ light quarks and their anti-quarks, and the heavy quark $H$ and anti-quark $\bar{H}$. For $a=H, \bar{H}$ we define $f_{a / p}^{N}(x, \mu)=0$ in the first term on the right hand side. The index $b$ runs over only the gluon and light quarks. At order $\alpha_{s}$, the only nonvanishing $A_{H b}$ is $A_{H g}$. Thus we may say that the $H$ distribution arises perturbatively from $g \rightarrow H \bar{H}$.

The $\alpha_{s}^{1}$ terms in the perturbative expansion of the $A_{a b}$ vanish at $\mu=M_{N+1}$. Thus one derives the simple matching conditon [12]: $f_{a / p}^{N+1}\left(x, M_{N+1}\right)=f_{a / p}^{N}\left(x, M_{N+1}\right)+\mathcal{O}\left(\alpha_{s}^{2}\right)$. The analogous connection is known at order $\alpha_{s}^{2}$ but we do not repeat it here.]

\section{Why is there an ambiguity?}

When we construct the hard scattering cross section in the presence of heavy quarks, there is a factorization ambiguity that is not present in the case of light quarks. Let us see why. Consider two enormously simplified examples that illustrate the principle.

First, suppose that there are only gluons and one light quark $L$ with mass $M_{L} \lesssim \Lambda$. Suppose, in addition, that the light quark is its own anti-particle, so that a quark $L$ and the anti-quark $\bar{L}$ are the same particle. Both the gluon and the light quark are considered to be active partons. In order to simplify the notation, let us take a moment $\int d x x^{n} F_{2}(x, Q)$ of $F_{2}$, so that we get a factorization formula involving the corresponding moments of $\widehat{F}_{2}$ and of the parton distributions. The dependence on the moment number will not be indicated. To further simplify the notation, let us set the factorization and renormalization scale $\mu$ to Q. Then

$$
F_{2}(Q) \sim \widehat{F}_{2}\left(L, \alpha_{s}(Q)\right) f_{L / p}(Q)+\widehat{F}_{2}\left(g, \alpha_{s}(Q)\right) f_{g / p}(Q) .
$$

Now $F_{2}$ is an observable, so its definition is fixed. We have defined the parton distribution functions, and the two parton distribution functions are independent. Thus this equation all but fixes the definition of $\widehat{F}_{2}\left(L, \alpha_{s}(Q)\right)$ and $\widehat{F}_{2}\left(g, \alpha_{s}(Q)\right)$. The only possible modification would be to add terms proportional to powers of $M_{L}^{2} / Q^{2}$ to $\widehat{F}_{2}\left(a, \alpha_{s}(Q)\right)$, at the cost of subtracting the same terms from the power suppressed remainder, $\mathcal{O}\left(\Lambda^{2} / Q^{2}\right)$, in the factorization formula (Eq. (11)). The simplest solution, which is uniformly adopted, is not to allow a $M_{L}^{2} / Q^{2}$ dependence in $\widehat{F}_{2}\left(a, \alpha_{s}(Q)\right)$.

Now suppose that there are only gluons and one (self-conjugate) heavy quark $H$ with mass $M_{H} \gg \Lambda$. Then in the scheme in which both the gluon and the heavy quark are

\footnotetext{
${ }^{1}$ At order $\alpha_{s}^{2}$, the matching at $\mu=M_{N+1}$ is no longer continous in general [8].
} 
considered to be active partons we have

$$
F_{2}(Q) \sim \widehat{F}_{2}\left(H, M_{H} / Q, \alpha_{s}(Q)\right) f_{H / p}(Q)+\widehat{F}_{2}\left(g, M_{H} / Q, \alpha_{s}(Q)\right) f_{g / p}(Q) .
$$

Here the $\widehat{F}_{2}\left(a, M_{H} / Q, \alpha_{s}(Q)\right)(\mathrm{a}=\mathrm{g}, \mathrm{H})$ depend on the heavy quark mass and we cannot move terms of order $M_{H}^{2} / Q^{2}$ into the power suppressed corrections because only terms of order $\Lambda^{2} / Q^{2}$ are allowed there. Thus it seems that we have no freedom. But we do: $f_{H / p}(Q)$ and $f_{g / p}(Q)$ are not independent. Since, according to Eq. (2), heavy quarks evolve from gluons, we have a relation of the form

$$
f_{H / p}(Q)=V_{H / g}\left(\ln \left(Q / M_{H}\right), \alpha_{s}(Q)\right) f_{g / p}(Q) .
$$

Here $V$ has a perturbative expansion that is obtained by solving the evolution equation. The first term has the form $V \sim \alpha_{s} \gamma \ln (Q / M)$ where $\gamma$ is a constant. Using Eq. (5) in Eq. (4) we obtain

$$
F_{2}(Q) \sim\left\{\widehat{F}_{2}\left(H, M_{H} / Q, \alpha_{s}(Q)\right) V_{H / g}\left(\ln \left(Q / M_{H}\right), \alpha_{s}(Q)\right)+\widehat{F}_{2}\left(g, M_{H} / Q, \alpha_{s}(Q)\right)\right\} f_{g / p}(Q) .
$$

Evidently, there is some freedom to move pieces from the first term in braces to the second. There is a constraint. For $M_{H} / Q \rightarrow 0$, it is possible to neglect $M_{H}$ in the calculation of the $\widehat{F}_{2}$. On the other hand, $V\left(\ln \left(Q / M_{H}\right), \alpha_{s}(Q)\right)$ does not have a smooth $M_{H} / Q \rightarrow 0$ limit. This is not a problem in applications because by solving the evolution equation one sums the leading logarithms $\left[\alpha_{s} \ln \left(Q / M_{H}\right)\right]^{n}$ in $V$. It is important not to undo this summation. Thus the factorization scheme that we adopt should have the property that the functions $\widehat{F}_{2}\left(a, M_{H} / Q, \alpha_{s}(Q)\right)$ have a finite limit as $M_{H} / Q \rightarrow 0$. This still leaves us the option of adding a term like $c \times\left(M_{H}^{2} / Q^{2}\right)$ to $\widehat{F}_{2}\left(H, M_{H} / Q, \alpha_{s}(Q)\right)$ and subtracting $c \times\left(M_{H}^{2} / Q^{2}\right) V$ from $\widehat{F}_{2}\left(g, M_{H} / Q, \alpha_{s}(Q)\right)$.

Suppose now that we have calculated $\widehat{F}_{2}\left(g, M_{H} / Q, \alpha_{s}(Q)\right)$ and $\widehat{F}_{2}\left(H, M_{H} / Q, \alpha_{s}(Q)\right)$ in some convenient prescription - for example the prescription analyzed in [9] based on "onshell" heavy quarks. Then we could define a new prescription with

$$
\widehat{F}_{2}^{\text {new }}\left(H, M_{H} / Q, \alpha_{s}(Q)\right)=\widehat{F}_{2}^{\text {old }}\left(H, 0, \alpha_{s}(Q)\right)
$$

and

$$
\begin{aligned}
& \widehat{F}_{2}^{\text {new }}\left(g, M_{H} / Q, \alpha_{s}(Q)\right)=\widehat{F}_{2}^{\text {old }}\left(g, M_{H} / Q, \alpha_{s}(Q)\right) \\
& \quad+\left\{\widehat{F}_{2}^{\text {old }}\left(H, M_{H} / Q, \alpha_{s}(Q)\right)-\widehat{F}_{2}^{\text {old }}\left(H, 0, \alpha_{s}(Q)\right)\right\} \times V_{H / g}\left(\ln \left(Q / M_{H}\right), \alpha_{s}(Q)\right) .
\end{aligned}
$$

In the following subsection, we shall give a prescription [9] based on this observation.

\section{A prescription for resolving the ambiguity}

Having seen the main idea, let us put the parton indices and the momentum fraction variables back. Suppose that there are $N+1$ quark flavors that we consider to be active. Let the 
heaviest active quark, quark $N+1$, be labelled $H$. Suppose that quark $H$ has a mass that is large compared to the hadronic mass scale $\Lambda$. In the $N+1$ flavor scheme the factorization equation is

$$
F_{2}(x, Q) \sim \int_{x}^{1} d \xi \sum_{a} f_{a / p}(\xi, \mu) \widehat{F}_{2}\left(a, x / \xi, M_{H} / Q, \mu / Q, \alpha_{s}(\mu)\right)
$$

where the sum over $a$ includes $a=H$ and $a=\bar{H}$.

The parton distributions for the gluon and $N+1$ quark flavors are obtained from the distributions in the scheme with only $N$ quark flavors. First we use the perturbative matching relation (Eq. (2)) at a scale near $\mu=M_{H}$, then we use the evolution equations to give the distribution functions at scale $\mu$. Since there are more output functions than input, we obtain a perturbative relation giving the heavy quark distribution functions at scale $\mu$ in terms of the light quark and gluon distribution functions at the same scale. This relation has the form

$$
f_{H / p}(\xi, \mu)=\int_{\xi}^{1} \frac{d \tau}{\tau} \sum_{a \neq H \bar{H}} f_{a / p}(\tau, \mu) V_{H / a}\left(\xi / \tau ; \mu / M_{H}, \alpha_{s}(\mu)\right)
$$

with an analogous equation for the heavy anti-quark $\bar{H}$. Inserting this relation into Eq. (9), we have

$$
F_{2}(x, Q) \sim \int_{x}^{1} d \xi \sum_{a \neq H, \bar{H}} f_{a / p}(\xi, \mu) T_{a}\left(x / \xi, M_{H} / Q, \mu / Q, \alpha_{s}(\mu)\right)
$$

where

$$
\begin{aligned}
& T_{a}(z,\left.M_{H} / Q, \mu / Q, \alpha_{s}(\mu)\right)=\widehat{F}_{2}\left(a, z, M_{H} / Q, \mu / Q, \alpha_{s}(\mu)\right) \\
& \quad+\int_{z}^{1} d \lambda \widehat{F}_{2}\left(H, z / \lambda, M_{H} / Q, \mu / Q, \alpha_{s}(\mu)\right) V_{H / a}\left(\lambda, \mu / M_{H}, \alpha_{s}(\mu)\right) \\
& \quad+\int_{z}^{1} d \lambda \widehat{F}_{2}\left(\bar{H}, z / \lambda, M_{H} / Q, \mu / Q, \alpha_{s}(\mu)\right) V_{\bar{H} / a}\left(\lambda, \mu / M_{H}, \alpha_{s}(\mu)\right) .
\end{aligned}
$$

We see that we have the same situation as in the simple example given earlier. Taking $\mu$ to be of order $Q$, one can shift contributions of order $M_{H}^{2} / Q^{2}$ between the hard scattering functions $\widehat{F}_{2}$ for $H$ and $\bar{H}$ and the corresponding functions for the light quarks and the gluon while keeping the functions $T$ unchanged for each light quark and gluon flavor $a$ and without ruining the property that all of the functions $\widehat{F}_{2}$ have finite $M_{H} / Q \rightarrow 0$ limits.

This freedom can be exploited to make the calculation of the functions $\hat{F}_{2}$ simpler. In particular, we can adopt a prescription proposed by Collins [9]:

Simplified ACOT (S-ACOT) prescription. Set $M_{H}$ to zero in the calculation of the hard scattering functions $\widehat{F}_{2}$ for incoming heavy quarks.

This observation tremendously simplifies the calculation of $\widehat{F}_{2}$ for $a=H$ as it reduces to that of the light-quark result. For example, at order $\alpha_{s}^{1}$, the $\widehat{F}_{2}$ functions for heavy quarks 
and light quarks are independent of $M_{H}$, and the calculation reduces to that of the simple massless result. The $\alpha_{s}^{1}$ gluon contribution to $\widehat{F}_{2}$ acquires an $M_{H}$ dependence when the gluon couples to a heavy quark loop, which is probed by the virtual photon $(g \gamma \rightarrow H \bar{H})$.

Note that the hard scattering functions $\widehat{F}_{2}$ obey a renormalization group equation that is different from the standard renormalization group equation that applies in the case of light flavors only. To see why this is so, imagine that the $\widehat{F}_{2}$ functions obeyed the usual renormalization equation and that $\widehat{F}_{2}\left(H, z / \lambda, M_{H} / Q, \mu / Q, \alpha_{s}(\mu)\right)$ were independent of $M_{H}$ at some fixed value of $\mu$. Then $\widehat{F}_{2}$ for $a=H, \bar{H}$ would depend on $M_{H}$ at other values of $\mu$ because the standard renormalization group equation mixes the heavy and light flavors and would mix $M_{H}$ dependence into $\widehat{F}_{2}\left(H, z / \lambda, M_{H} / Q, \mu / Q, \alpha_{s}(\mu)\right)$.

This Simplified ACOT prescription has the advantage of being simple to state. In addition, its calculational simplicity could be crucial for applying variable-flavor-number prescriptions with mass in analyses that go beyond first order in $\alpha_{s}$.

\section{The ACOT and S-ACOT prescriptions at first order}

In this section, we analyze the ACOT prescription and its simplified version, the S-ACOT prescription, at order $\alpha_{s}^{1}$. We consider neutral current deeply inelastic scattering from a proton target in a $Q^{2}$ regime in which quarks $1, \ldots, N$ can be considered as light while a single quark, $H$, is considered to be heavy or light depending on the value of $Q^{2}$. In order to keep the analysis simple, contributions from all heavier quarks are ignored. We further simplify the problem by supposing that the vector boson current that probes the proton couples only to the heavy quark $H$ and its anti-quark $\bar{H}$, but not to the lighter quarks or gluons. (In a crude approximation, this is like taking ordinary deeply inelastic scattering but demanding that $H$ appear in the final state.) Let $F$ be one of the structure functions $F_{1}$, $F_{2} / x$ or $F_{3}$ for our special vector boson. We choose the factorization and renormalization scales $\mu$ equal to $Q$. We write the factorization formula for $F$ in the shorthand notation

$$
F=\sum_{a} \widehat{F}_{a} \otimes f_{a / p}+\mathcal{O}\left(\Lambda^{2} / Q^{2}\right)
$$

where the sum runs over all partons $a$ including $H$ and $\bar{H}$. The $\otimes$ denotes a convolution, so that Eq. (13) means

$$
F(x, Q)=\sum_{a} \int \frac{d \xi}{\xi} \widehat{F}_{a}\left(x / \xi, Q ; \alpha_{s}(Q)\right) f_{a / p}(\xi, \mu)+\mathcal{O}\left(\Lambda^{2} / Q^{2}\right) .
$$

The functions $\widehat{F}_{a}$ in Eq. (13) are the hard scattering functions. They have an expansion in powers of $\alpha_{s}$. Keeping the first two terms in this expansion gives

$$
\begin{aligned}
F= & \widehat{F}_{H}^{(0)} \otimes f_{H / p}+\widehat{F}_{\bar{H}}^{(0)} \otimes f_{\bar{H} / p} \\
& +\widehat{F}_{g}^{(1)} \otimes f_{g / p}+\widehat{F}_{H}^{(1)} \otimes f_{H / p}+\widehat{F}_{\bar{H}}^{(1)} \otimes f_{\bar{H} / p} \\
& +\mathcal{O}\left(\alpha_{s}^{2}\right)+\mathcal{O}\left(\Lambda^{2} / Q^{2}\right),
\end{aligned}
$$


where $\widehat{F}_{a}^{(n)}$ is the order $\alpha_{s}^{n}$ contribution to $\widehat{F}_{a}$.

In the ACOT prescription, we choose the order zero hard scattering function for a heavy quark to be

$$
\widehat{F}_{H, \mathrm{ACOT}}^{(0)}=F_{H}^{(0)}\left(M_{H}\right),
$$

where $F_{H}^{(0)}\left(M_{H}\right)$ is the calculated structure function for scattering from an initial state heavy quark of mass $M_{H}$ that is on its mass-shell. (To be precise, the heavy quark transverse momentum is taken to be zero, and the structure functions $F_{1}, F_{2} / x, F_{3}$ are extracted from the tensor $W^{\mu \nu}$ using the usual formula but with the quark momentum $k^{\mu}$ replaced by a light-like vector $\tilde{k}^{\mu}=k^{\mu}-\left[M_{H}^{2} /(2 u \cdot k)\right] u^{\mu}$, where $u^{\mu}$ is a light-like reference vector in the plane of $q^{\mu}$ and $k^{\mu}$.) The function $F_{H}^{(0)}\left(M_{H}\right)$ is rather complicated (see Ref. 田, 13, 14]), so we do not reproduce it here. The definition of $\widehat{F}_{\bar{H}, \mathrm{ACOT}}^{(0)}$ for a heavy anti-quark is analogous.

The order $\alpha_{s}$ gluon hard scattering function in Eq. (15) has three pieces:

$$
\widehat{F}_{g, \mathrm{ACOT}}^{(1)}=F_{g}^{(1)}\left(M_{H}\right)-F_{H}^{(0)}\left(M_{H}\right) \otimes f_{H / g}^{(1)}\left(M_{H}\right)-F_{\bar{H}}^{(0)}\left(M_{H}\right) \otimes f_{\bar{H} / g}^{(1)}\left(M_{H}\right) .
$$

Here $F_{g}^{(1)}\left(M_{H}\right)$ is the calculated structure function for scattering from an initial state massless gluon that is on its mass-shell, using graphs with a heavy quark loop and a heavy anti-quark loop. The function $f_{H / g}^{(1)}\left(M_{H}\right)$ is the calculated $\alpha_{s}^{1}$ contribution to the distribution of heavy quarks in an on-mass-shell gluon. Similarly, $f_{\bar{H} / g}^{(1)}\left(M_{H}\right)$ is the calculated $\alpha_{s}^{1}$ contribution to the distribution of heavy anti-quarks in an on-mass-shell gluon. Both functions are given by

$$
f_{H / g}^{(1)}(\xi, Q)=f_{\bar{H} / g}^{(1)}(\xi, Q)=\frac{\alpha_{s}(Q)}{2 \pi} \ln \frac{Q^{2}}{M_{H}^{2}} P_{q g}^{(1)}(\xi),
$$

where $P_{q / g}$ is the usual gluon $\rightarrow$ quark splitting function $P_{q g}(\xi)=T_{F}\left(\xi^{2}+(1-\xi)^{2}\right)$.

The first order hard scattering function for a heavy quark has a structure similar to that of the corresponding function for a gluon,

$$
\widehat{F}_{H, \mathrm{ACOT}}^{(1)}=F_{H}^{(1)}\left(M_{H}\right)-F_{H}^{(0)}\left(M_{H}\right) \otimes f_{H / H}^{(1)}\left(M_{H}\right) .
$$

Here $F_{H}^{(1)}\left(M_{H}\right)$ is the order $\alpha_{s}^{1}$ contribution to the structure function for scattering from an initial state heavy quark that is on its mass-shell, as given in 13 . The function $f_{H / H}^{(1)}\left(M_{H}\right)$ is the calculated $\alpha_{s}^{1}$ contribution to the distribution of heavy quarks in an on-mass-shell heavy quark,

$$
f_{H / H}^{(1)}(\xi, Q)=C_{F} \frac{\alpha_{s}(Q)}{2 \pi}\left[\frac{1+\xi^{2}}{1-\xi}\left\{\ln \left(\frac{Q^{2}}{(1-\xi)^{2} M_{H}^{2}}\right)-1\right\}\right]_{+},
$$

where the + subscript denotes the usual prescription,

$$
\int_{0}^{1} d \xi f(\xi)[F(\xi)]_{+}=\int_{0}^{1} d \xi\{f(\xi)-f(1)\} F(\xi) .
$$

This result can be calculated easily from the definition [1] of $\overline{\mathrm{MS}}$ parton distribution functions or it can be extracted from the ACOT subtraction terms in 13.

This defines the ACOT prescription at order $\alpha_{s}^{1}$. The prescription has two important properties. 
- Property 1. For $Q / M \rightarrow \infty$, the hard scattering functions in Eq. (15) approach the hard scattering functions of the ZM-VFN prescription, in which the heavy quark $H$ is taken as a parton with zero mass.

To be specific, we have the relations:

$$
\begin{aligned}
\widehat{F}_{H, \mathrm{ZM}-\mathrm{VFN}}^{(0)} & =F_{H}^{(0)}(0), \\
\widehat{F}_{g, \mathrm{ZM}-\mathrm{VFN}}^{(1)} & =F_{g}^{(1)}(0)-F_{H}^{(0)}(0) \otimes f_{H / g}^{(1)}(0)-F_{\bar{H}}^{(0)}(0) \otimes f_{\bar{H} / g}^{(1)}(0), \\
\widehat{F}_{H, \mathrm{ZM}-\mathrm{VFN}}^{(1)} & =F_{H}^{(1)}(0)-F_{H}^{(0)}(0) \otimes f_{H / H}^{(1)}(0) .
\end{aligned}
$$

We should note that in a calculation with $M_{H}=0$, there are infrared divergences. Thus the calculations are performed in $4-2 \epsilon$ dimensions of space-time and $1 / \epsilon$ poles appear. The pole terms cancel in Eq. (22). Instead of using dimensional regulation and taking $\epsilon \rightarrow 0$, one could use an infrared regulator mass $m$ and let $m \rightarrow 0$.

- Property 2. For $Q$ of order $M_{H}$, the structure function is that of the fixed-flavor-number prescription, up to corrections of order $\alpha_{s}^{2}$.

In the fixed-flavor-number prescription we have

$$
F=F_{g}^{(1)}\left(M_{H}\right) \otimes f_{g / p}+\mathcal{O}\left(\alpha_{s}^{2}\right)+\mathcal{O}\left(\Lambda^{2} / Q^{2}\right) .
$$

Property 2 follows because when the factorization scale, $\mu=Q$, is of order $M$, the heavy quark distribution function is given by the perturbative formula

$$
f_{H / p}=f_{H / g}^{(1)} \otimes f_{g / p}+\mathcal{O}\left(\alpha_{s}^{2}\right) .
$$

First of all, since $f_{H / p}$ is of order $\alpha_{s}$, the terms

$$
\widehat{F}_{H}^{(1)} \otimes f_{H / p}+\widehat{F}_{\bar{H}}^{(1)} \otimes f_{\bar{H} / p}
$$

in Eq. (15) are of order $\alpha_{s}^{2}$ and can be dropped. This leaves

$$
\begin{aligned}
F & =F_{H}^{(0)}\left(M_{H}\right) \otimes f_{H / p}+F_{\bar{H}}^{(0)}\left(M_{H}\right) \otimes f_{\bar{H} / p} \\
& +\left[F_{g}^{(1)}\left(M_{H}\right)-F_{H}^{(0)}\left(M_{H}\right) \otimes f_{H / g}^{(1)}\left(M_{H}\right)-F_{\bar{H}}^{(0)}\left(M_{H}\right) \otimes f_{\bar{H} / g}^{(1)}\left(M_{H}\right)\right] \otimes f_{g / p} \\
& +\mathcal{O}\left(\alpha_{s}^{2}\right)+\mathcal{O}\left(\Lambda^{2} / Q^{2}\right) .
\end{aligned}
$$

Inserting Eq. (24) into Eq. (26), we obtain Eq. (23).

We can summarize properties 1 and 2 by saying that the ACOT prescription interpolates between the zero-mass prescription for $M_{H} \ll Q$ and the fixed-flavor-number prescription for $M_{H} \sim Q$. 
What about the simplified ACOT prescription? We use the same formulas as for the ACOT prescription, but set $M_{H}=0$ in the hard scattering functions for heavy quarks:

$$
\begin{aligned}
\widehat{F}_{H, \mathrm{~S}-\mathrm{ACOT}}^{(0)} & =F_{H}^{(0)}(0), \\
\widehat{F}_{g, \mathrm{~S}-\mathrm{ACOT}}^{(1)} & =F_{g}^{(1)}\left(M_{H}\right)-F_{H}^{(0)}(0) \otimes f_{H / g}^{(1)}\left(M_{H}\right)-F_{\bar{H}}^{(0)}(0) \otimes f_{\bar{H} / g}^{(1)}\left(M_{H}\right), \\
\widehat{F}_{H, \mathrm{~S}-\mathrm{ACOT}}^{(1)} & =F_{H}^{(1)}(0)-F_{H}^{(0)}(0) \otimes f_{H / H}^{(1)}(0) .
\end{aligned}
$$

Repeating the derivation just given, we see that properties 1 and 2 hold for the S-ACOT prescription. That is, the S-ACOT prescription also interpolates between the zero-mass prescription for $M_{H} \ll Q$ and the fixed-flavor-number prescription for $M_{H} \sim Q$. However the S-ACOT prescription has the advantage that the functions $\widehat{F}_{H}$ are easier to calculate.

\section{Comparison of different prescriptions}

In this section, we investigate how well the matching properties among the different prescriptions work. In the plots presented, the masses and couplings have been fixed to be consistent with the values used in the CTEQ4L/M fits, i.e. $m_{c}=1.6 \mathrm{GeV}, m_{b}=5 \mathrm{GeV}$ [15]. Below the bottom threshold $\mathrm{n}_{\mathrm{f}}=4$ active flavors are used for $\alpha_{s}$, and the scale has been chosen as $\mu=Q$.

\subsection{Parton distribution matching}

In the threshold region, the structure function calculated in the S-ACOT prescription matches that calculated in the FFN prescription, in which the heavy quark does not appear as a parton. This matching, Property 2, results from the fact that the heavy quark distribution function $f_{H / p}(x, \mu)$, matches the approximate function

$$
\widetilde{f}_{H / p}(\mu)=f_{H / g}^{(1)}(\mu) \otimes f_{g / p}(\mu)
$$

Both functions vanish at $\mu=M_{H}$ and, for $\mu>M_{H}$, the difference between them is of order $\alpha_{s}^{2}$. In this subsection, we study the quality of this matching.

In Figs. $1-3$ we plot $f_{H / p}(x, \mu)$ and $\widetilde{f}_{H / p}(x, \mu)$ for the case of the charm quark. In Fig. 1 , we use CTEQ4L parton distributions, which are based on lowest order evolution. Fig. 1 reveals that the matching works very well when the order $\alpha_{s}^{1}$ evolution kernel is used for $f_{H / p}(x, \mu)$ and the order $\alpha_{s}^{1}$ perturbative expression (Eq. (28)) is used for $\tilde{f}$. In Fig. 2, we use CTEQ4M parton distributions, which are based on NLO evolution. We observe a mismatch because the full NLO evolution kernel is used for $f_{H / p}$ while the order $\alpha_{s}^{1}$ perturbative expansion is used for $\tilde{f}$. The difference between $f$ and $\tilde{f}$ is of order $\alpha_{s}^{2}$, but this difference is numerically quite large. Finally, we see in Fig. 3 that a close match is restored when the NLO evolution kernel is used for $f$ while $\tilde{f}$ is defined using a modified version of Eq. (28) in which we replace 


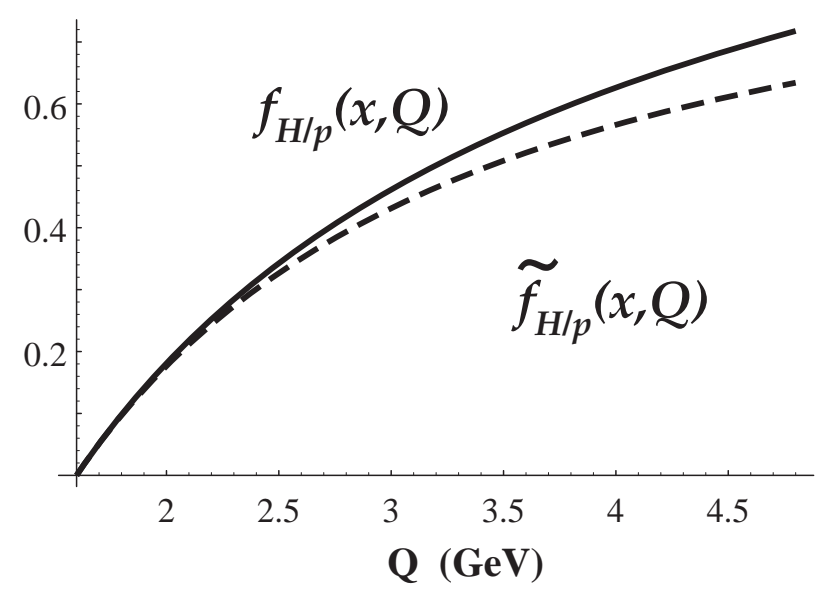

Figure 1: CTEQ4L charm quark density $f_{H / p}(x, Q)$ and the approximate form $\tilde{f}_{H / p}(x, Q)$, Eq. (28), at $x=0.05$ as function of $Q$. Since Eq. (28) is an order $\alpha_{s}^{1}$ perturbative approximation and the evolution kernel for the CTEQ4L parton distribution is also order $\alpha_{s}^{1}$, these curves match closely at threshold.

the leading order $g \rightarrow H$ evolution kernel in Eq. (18) by the next-to-leading order $g \rightarrow H$ evolution kernel.[?

We can draw two conclusions. First, the threshold matching discussed in the previous section will work order by order for the perturbation expansion. [] Second, the order $\alpha_{s}^{2}$ terms in the evolution kernel are quite large, so that the leading order calculations illustrated in this paper may not be sufficient for obtaining accurate predictions.f

\subsection{Structure function matching}

In this subsection, we examine predictions for $F_{2}^{c}(x, Q)$, which we define here to be the contribution to $F_{2}(x, Q)$ from graphs in which the current couples to a charm quark. We compare $F_{2}^{c}(x, Q)$ calculated with the S-ACOT prescription at order $\alpha_{s}$ with that calculated with the original ACOT prescription, the ZM-VFN prescription in which the charm quark can appear as a parton but has zero mass, and the FFN prescription in which the charm

\footnotetext{
${ }^{2}$ For the NLO perturbative expansion, we have included the $\alpha_{s}^{2}$ splitting kernels, $P_{j / i}^{(2)}(\xi)$. For simplicity, we have ignored iterated terms such as $P_{k / j}^{(1)} \otimes P_{j / i}^{(1)}$ which contribute as $\ln ^{2}\left(\mu / M_{H}\right)$, and hence only play a role away from threshold.

${ }^{3}$ At order $\alpha_{s}^{2}$, Property 2 is still preserved even though the matching conditions on the parton distribution functions are modified. The $\alpha_{s}^{2}$ matching conditions shift the evolved parton distribution functions $f_{H / p}$, but they also shift the "perturbative" parton distribution functions $\widetilde{f}_{H / p}$ leaving the difference unchanged up to order $\alpha_{s}^{3}$.

${ }^{4}$ One might ask whether retaining the heavy quark mass in the kinematics (in the spirit of the "slowrescaling" correction) might prove beneficial. The answer is that as long as the order of the parton distribution functions and the hard scattering coefficients are matched, this simply amounts to a shuffling of $M_{H} / Q$ terms between the quark and gluon initiated terms, $c f .$, Eq. (5).
} 


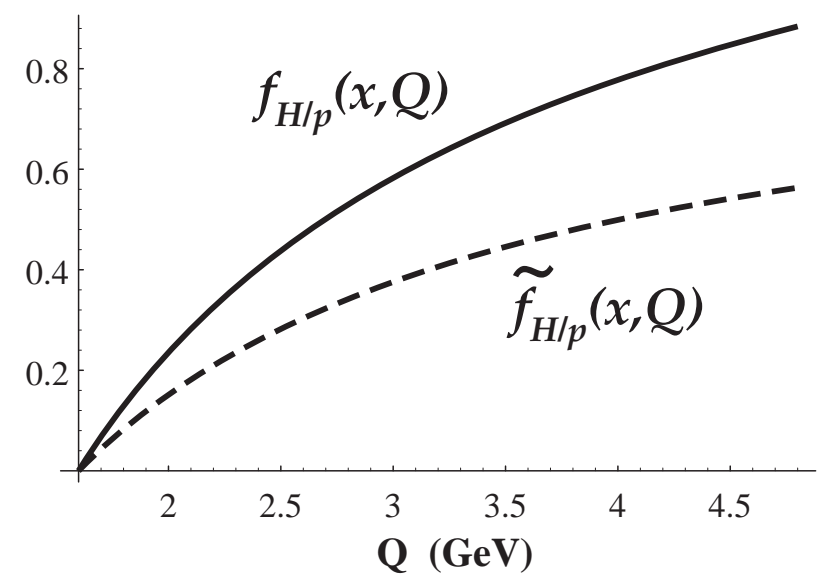

Figure 2: CTEQ4M charm quark density $f_{H / p}(x, Q)$ and the approximate form $\tilde{f}_{H / p}(x, Q)$, Eq. (28), at $x=0.05$ as function of $Q$. Since Eq. (28) is an order $\alpha_{s}^{1}$ perturbative approximation while the evolution kernel for the CTEQ4M parton distribution includes order $\alpha_{s}^{2}$ terms, these curves do not match closely at threshold.

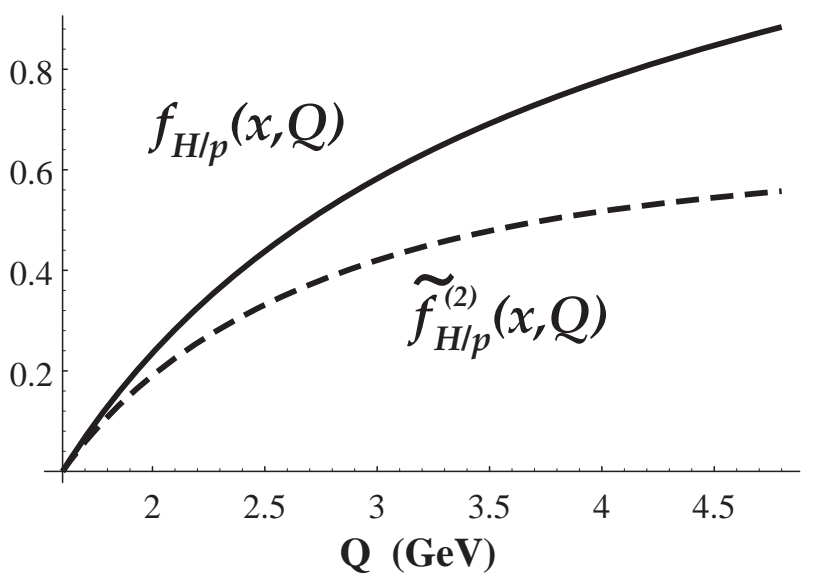

Figure 3: CTEQ4M charm quark density $f_{H / p}(x, Q)$ and an approximate form $\tilde{f}_{H / p}^{(2)}(x, Q)$ at $x=0.05$ as function of $Q$. The approximate form is based on an analogue of Eq. (28) in which appropriate order $\alpha_{s}^{2}$ terms are added. Since the calculation of $\tilde{f}_{H / p}^{(2)}(x, Q)$ uses the second order evolution kernel used for the CTEQ4M parton distributions, these curves match closely at threshold. 

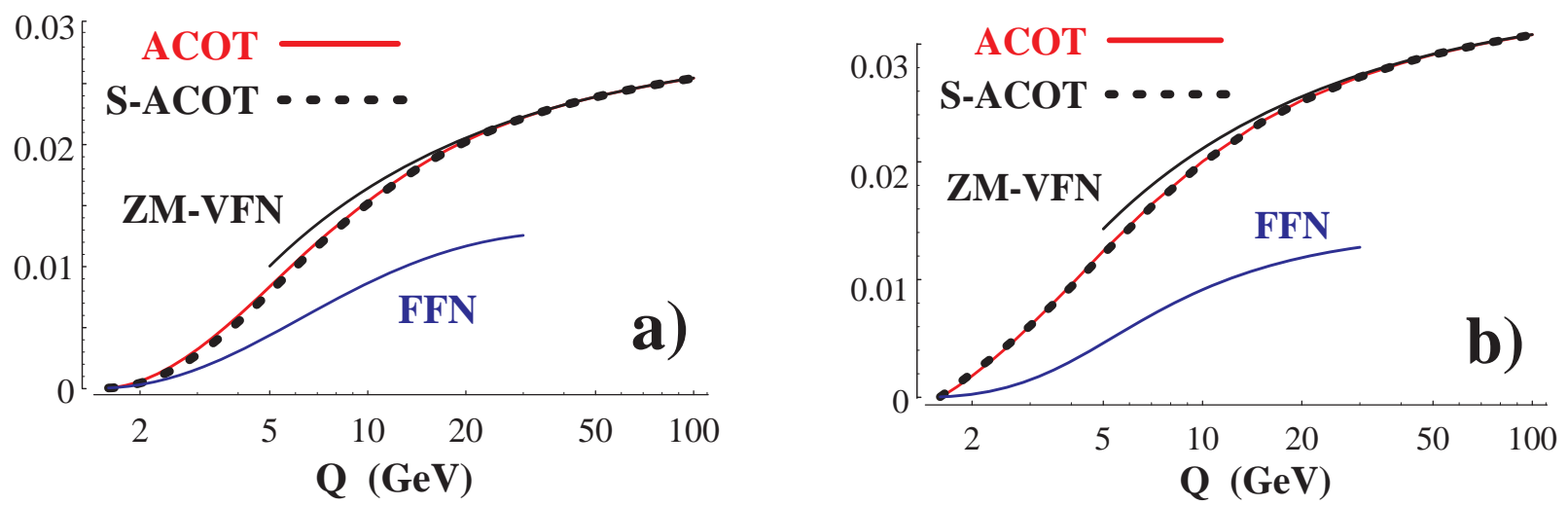

Figure 4: $\quad F_{2}^{c}$ for $x=0.1$ as a function of $Q$ as calculated using the ZM-VFN, FFN, ACOT, and S-ACOT prescriptions. The hard scattering coefficients are calculated to order $\alpha_{s}^{1}$. Plot a) uses CTEQ4L parton densities and Plot b) uses CTEQ4M parton densities.

quark has its proper mass but does not appear as a parton. For simplicity, we take $\mu=Q$. In our calculations, we evaluate the hard scattering coefficients $\widehat{F}$ at order $\alpha_{s}$. Thus for the ZM-VFN, ACOT, and S-ACOT prescriptions, the terms displayed in Eq. (15) are included. The functions $\widehat{F}$ are given by Eq. (22) for ZM-VFN, by Eqs. (16, 17, 19) for ACOT, and by Eq. (27) for S-ACOT. For the FFN prescription, there is only one term at order $\alpha_{s}$, as displayed in Eq. (23).

In Fig. 4 we show $F_{2}^{c}(x, Q)$ as a function of $Q$ for $x=0.1$. Then in Fig. 5 we show $F_{2}^{c}(x, Q)$ as a function of $Q$ for $x=0.001$. In each case we display results using both the CTEQ4L and CTEQ4M parton distributions.

When we use the CTEQ4L parton distributions, we notice that there is a close match between the S-ACOT result and the FFN result near $Q=m_{c}$. Based on the results of the previous subsection, we expect this matching to be degraded when we use CTEQ4M parton distributions because of the important role played by the order $\alpha_{s}^{2}$ term in the evolution kernel that is not matched in the lowest order calculation of the hard scattering function. This degradation is seen in the figures.

In the asymptotic regime, $Q \gg M$, we find the S-ACOT result approximates the ZMVFN result, as expected.

We observe that the ACOT and S-ACOT prescriptions are effectively identical throughout the kinematic range. There is a slight difference in the threshold region, but this is small in comparison to the size of the $\mu$-variation (not shown). Hence the difference between the ACOT and S-ACOT results is of no physical consequence. The fact that the ACOT and S-ACOT prescriptions match extremely well throughout the full kinematic range provides explicit numerical verification that the S-ACOT prescription fully contains the physics. 

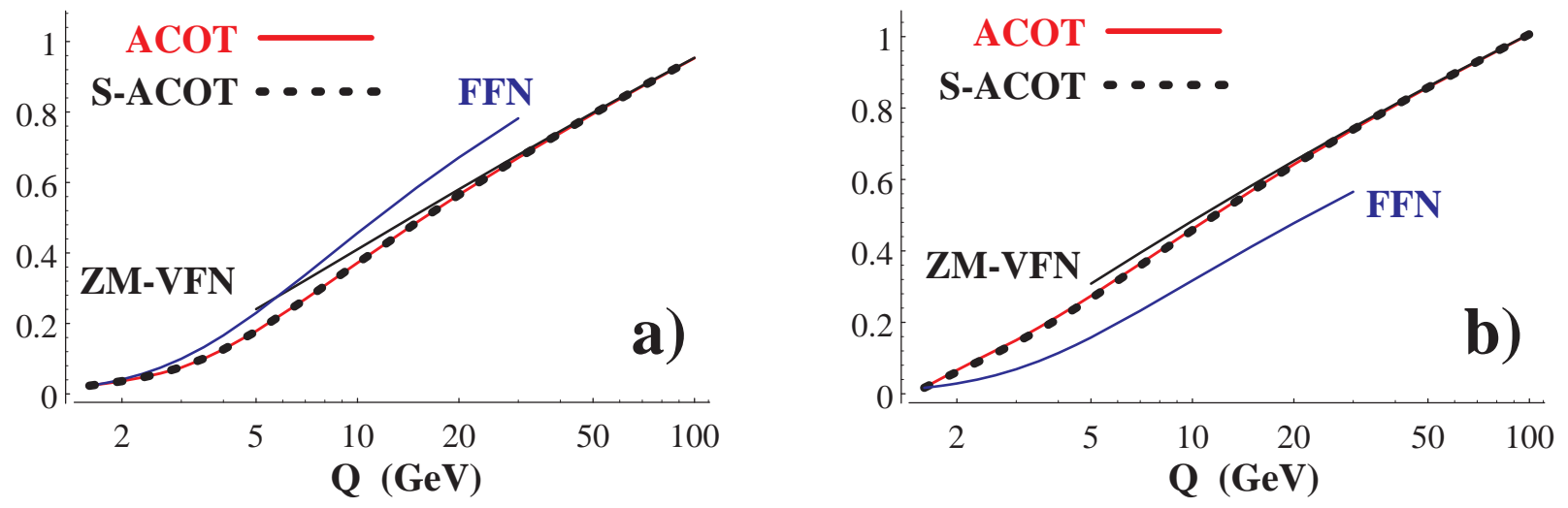

Figure 5: $\quad F_{2}^{c}$ for $x=0.001$ as a function of $Q$ as calculated using the ZM-VFN, FFN, ACOT, and S-ACOT prescriptions. The hard scattering coefficients are calculated to order $\alpha_{s}^{1}$. Plot a) uses CTEQ4L parton densities and Plot b) uses CTEQ4M parton densities.

\section{Conclusions and outlook}

We have performed a numerical study of different prescriptions for dealing with the quark mass in heavy quark leptoproduction. We have seen that the simplest prescription [9], SACOT, is numerically equivalent to the earlier ACOT prescription [4].

The S-ACOT prescription is extensible order by order in $\alpha_{s}$. At $\mathcal{O}\left(\alpha_{s}^{1}\right)$ we already find a significant simplification in the S-ACOT prescription as compared with the ACOT prescription. We expect that this simplification becomes even more dramatic at higher orders.

We have not attempted to implement the S-ACOT prescription at order $\alpha_{s}^{2}$, but we note that the NLO corrections in the the FFN prescription have been calculated [2], and that the leading (collinear) logarithms of the type $\alpha_{s}^{i} \log ^{i}\left(Q^{2} / M_{H}^{2}\right)$ have been extracted in analytic form [8]. Thus the S-ACOT subtraction term can be constructed as well.

Finally, we emphasize that the choice of a prescription for dealing with quark masses in the hard scattering coefficients for deeply inelastic scattering is a separate issue from the choice of definition of the parton distribution functions. For all of the prescriptions discussed here, one uses the standard $\overline{\mathrm{MS}}$ definition of parton distributions.

\section{Acknowledgments}

We thank J.C. Collins, R.J. Scalise, R.S. Thorne, and W.-K. Tung for valuable discussions and we thank S. Kretzer for making his NLO code available to us. We thank the CERN Theory Division, Fermilab Theory Group, and the University of Oregon for their kind hospitality during the period in which part of this research was carried out. This work is supported by the U.S. Department of Energy, the Lightner-Sams Foundation, and in part by the EU TMR contract FMRX-CT98-0194 (DG 12 - MIHT). 


\section{References}

[1] R. M. Barnett, H. E. Haber, and D. E. Soper, Nucl. Phys. B306, 697 (1988).

[2] E. Laenen, S. Riemersma, J. Smith, and W. L. van Neerven, Nucl. Phys. B392, 229 (1993); Phys. Rev. D49, 5753 (1994) hep-ph/9308295].

[3] F. I. Olness and S. T. Riemersma, Phys. Rev. D51, 4746 (1995) hep-ph/9409208.

[4] F. I. Olness and W. K. Tung, Nucl. Phys. B308, 813 (1988); M. A. Aivazis, F. I. Olness, and W. K. Tung, Phys. Rev. D50, 3085 (1994) hep-ph/9312318; M. A. Aivazis, J. C. Collins, F. I. Olness, and W. K. Tung, Phys. Rev. D50, 3102 (1994) hep-ph/9312319.

[5] R. S. Thorne and R. G. Roberts, Phys. Rev. D57, 6871 (1998) hep-ph/9709442; Phys. Lett. B421, 303 (1998) hep-ph/9711223.

[6] H. L. Lai and W. K. Tung, Z. Phys. C74, 463 (1997) hep-ph/9701256.

[7] A. D. Martin, R. G. Roberts, W. J. Stirling, and R. S. Thorne, Eur. Phys. J. C4, 463 (1998) hep-ph/9803445.

[8] M. Buza, Y. Matiounine, J. Smith, R. Migneron, and W. L. van Neerven, Nucl. Phys. B472, 611 (1996) hep-ph/9601302; M. Buza, Y. Matiounine, J. Smith, and W. L. van Neerven, Phys. Lett. B411, 211 (1997) hep-ph/9707263; M. Buza, Y. Matiounine, J. Smith, and W. L. van Neerven, Eur. Phys. J. C1, 301 (1998) hep-ph/9612398; A. Chuvakin, J. Smith and, W. L. van Neerven, hep-ph/9910250.

[9] J. C. Collins, Phys. Rev. D58, 094002 (1998) hep-ph/9806259.

[10] J. C. Collins, F. Wilczek, and A. Zee, Phys. Rev. D18, 242 (1978).

[11] J. C. Collins and D. E. Soper, Nucl. Phys. B194, 445 (1982).

[12] J. C. Collins and W. K. Tung, Nucl. Phys. B278, 934 (1986).

[13] S. Kretzer and I. Schienbein, Phys. Rev. D56, 1804 (1997) hep-ph/9702296; Phys. Rev. D58, 094035 (1998) hep-ph/9805233]; Phys. Rev. D59, 054004 (1999) [hep-ph/9808375].

[14] C. R. Schmidt, hep-ph/9706496; J. Amundson, C. Schmidt, W. K. Tung, and X. Wang, MSU preprint, in preparation; J. Amundson, F. I. Olness, C. R. Schmidt, W. K. Tung, and X. Wang, FERMILAB-CONF-98-153-T, to be published in the proceedings of 6th International Workshop on Deep Inelastic Scattering and QCD (DIS 98), Brussels, Belgium, 4-8 Apr 1998.

[15] H. L. Lai et al., Phys. Rev. D55, 1280 (1997) hep-ph/9606399. 